UMIACS-TR-91-79

May 1991

CS-TR 2685

\title{
ERror ANALYSis of QR UPDATING With Exponential Windowing
}

\author{
G. W. STEWART*
}

\begin{abstract}
Exponential windowing is a widely used technique for suppressing the effects of old data as new data is added to a matrix. Specifically, given an $n \times p$ matrix $X_{n}$ and a "forgetting factor" $\beta \in(0,1)$, one works with the matrix $\operatorname{diag}\left(\beta^{n-1}, \beta^{n-2}, \ldots, 1\right) X_{n}$. In this paper we examine an updating algorithm for computing the $\mathrm{QR}$ factorization of $\operatorname{diag}\left(\beta^{n-1}, \beta^{n-2}, \ldots, 1\right) X_{n}$ and show that it is unconditionally stable in the presence of rounding errors.
\end{abstract}

* Department of Computer Science and Institute for Advanced Computer Studies, University of Maryland, College Park, MD 20742. This report is available by anonymous ftp from thales.cs.umd.edu in the directory pub/reports. This work was supported in part by the Air Force Office of Scientific Research under Contract AFOSR-87-0188. 


\section{Error Analysis of QR Updating with Exponential Windowing}

\section{G. W. Stewart*}

\section{Introduction}

In many application (e.g., signal processing, time series) one needs the QR factorization of an $n \times p$ matrix

$$
X_{n}=\left(\begin{array}{c}
x_{1}^{\mathrm{H}} \\
x_{2}^{\mathrm{H}} \\
\vdots \\
x_{n}^{\mathrm{H}}
\end{array}\right) \text {. }
$$

The rows of $X_{n}$ represent data that arrives at regular intervals, with $x_{1}^{\mathrm{H}}$ the oldest data and $x_{n}^{\mathrm{H}}$ the most recent.

If the series $x_{n}^{\mathrm{H}}$ is not stationary, it is necessary to suppress the older data so that they do not contaminate more recent information. One widely used method for accomplishing this is called exponential windowing. Let $\beta \in(0,1)$ be a "forgetting factor," and let

$$
D_{n}=\operatorname{diag}\left(\beta^{n-1}, \beta^{n-2}, \ldots, 1\right) .
$$

Instead of computing the QR factorization of $X_{n}$, one computes the QR factorization of $D_{n} X_{n}$; i.e., one computes

$$
D_{n} X_{n}=Q_{n} R_{n},
$$

where $Q_{n}$ has orthonormal columns and $R_{n}$ is upper triangular. The effect of exponential windowing is to weight $x_{i}^{\mathrm{H}}$ by $\beta^{n-i}$, so that it has less and less influence as $n$ increases.

* Department of Computer Science and Institute for Advanced Computer Studies, University of Maryland, College Park, MD 20742. This report is available by anonymous ftp from thales.cs. umd.edu in the directory pub/reports. This work was supported in part by the Air Force Office of Scientific Research under Contract AFOSR-87-0188. 
QR with Exponential Windowing

As a rule, only the R-factor $R_{n}$ in (1.1) is needed in applications. It can be computed efficiently by the following updating procedure. Let $R_{0}=0$. Given $R_{n}$, compute the QR decomposition:

$$
\left(\begin{array}{cc}
U_{n} & v_{n} \\
w_{n}^{\mathrm{H}} & \xi_{n}
\end{array}\right)\left(\begin{array}{l}
\beta R_{n} \\
x_{n+1}^{\mathrm{H}}
\end{array}\right)=\left(\begin{array}{c}
R_{n+1} \\
0
\end{array}\right) .
$$

Here

$$
\left(\begin{array}{cc}
U_{n} & v_{n} \\
w_{n}^{\mathrm{H}} & \xi_{n}
\end{array}\right)
$$

is unitary and $R_{n+1}$ is upper triangular. Is is easily seen that the sequence of triangular matrices $R_{n}$ so generated are the $\mathrm{R}$-factors of the matrices $X_{n}$. The details of this updating algorithm may be found in [1]. It requires $O\left(p^{2}\right)$ arithmetic operations.

Exponential windowing and updating allows us to look at the local behavior of an arbitrarily long sequence of data. However, the fact that $n$ is effectively unbounded raises the possibility that rounding error will accumulate to the point where it overwhelms the data. The purpose of this paper is to show that this does not happen: exponential windowing damps old rounding errors along with old data.

In the next section we will present the rounding error analysis. Although the results of this analysis are sufficient for practical purposes, it is clear that the bounds are an overestimate, at least asymptotically. Consequently, $\S 3$ is devoted to producing refined bounds.

Throughout this paper $\|X\|$ will denote the Frobenius norm defined by

$$
\|A\|^{2}=\sum_{i, j}\left|a_{i j}\right|^{2}
$$

All computations will be assumed to be in floating point arithmetic with rounding unit $\epsilon_{\mathrm{M}}$; i.e., $-\log \epsilon_{\mathrm{M}}$ is approximately the number of decimal digits carried in the computation.

\section{The Error Analysis}

Our error analysis will be a classical backward error analysis; that is, we will show that the computed $R_{n}$, whatever its accuracy, comes from very slightly perturbed data. The analysis begins with an a backward error analysis of the single update step (1.2). 
Theorem 2.1. Let $R_{n+1}$ denote the result of performing the update (1.2) in floating-point arithmetic with rounding unit $\epsilon_{\mathrm{M}}$. Then there is a unitary matrix of the form (1.3), a constant $K$ depending on $p$, a matrix $G$ and a vector $h^{\mathrm{H}}$ satisfying

$$
\left\|\left(\begin{array}{c}
G_{n} \\
h_{n}^{\mathrm{H}}
\end{array}\right)\right\| \leq K \epsilon_{\mathrm{M}}\left\|\left(\begin{array}{c}
\beta R_{n} \\
x_{n+1}^{\mathrm{H}}
\end{array}\right)\right\|
$$

such that

$$
\left(\begin{array}{cc}
U_{n} & v_{n} \\
w_{n}^{\mathrm{H}} & \xi_{n}
\end{array}\right)\left(\begin{array}{c}
\beta R_{n}+G_{n} \\
x_{n+1}+h_{n+1}^{\mathrm{H}}
\end{array}\right)=\left(\begin{array}{c}
R_{n+1} \\
0
\end{array}\right)
$$

A proof of this theorem may be found in [4, Ch.3, $\S \S 20-24]$.

The analysis of the updating algorithm with exponential windowing amounts to the recursive application of the bound (2.2). As is typical in backward roundingerror analyses, we let let quantities stand for their computed values. The results will be cast in terms of the augmented factorization

$$
\left(\begin{array}{c}
0 \\
D_{n} X_{n}
\end{array}\right)=\left(\begin{array}{c}
0 \\
Q_{n}
\end{array}\right) R_{n} .
$$

This factorization reflects the actual updating process in which we start with a zero matrix, imagined to lie above $X_{n}$, and form $R_{n}$ in it.

Theorem 2.2. Let

$$
\left\|R_{n}\right\| \leq \rho, \quad n=1,2, \ldots,
$$

so that $\rho$ is an upper bound for the norms of the computed $R_{n}$. Then there is a matrix

$$
\left(\begin{array}{c}
P_{n} \\
Q_{n}
\end{array}\right)
$$

with orthonormal columns and matrices $E_{n}$ and $F_{n}$ satisfying

$$
\left\|\left(\begin{array}{c}
E_{n} \\
F_{n}
\end{array}\right)\right\| \leq \frac{K \epsilon_{\mathrm{M}} \rho}{(1-\beta)\left(1-K \epsilon_{\mathrm{M}}\right)}
$$

such that

$$
\left(\begin{array}{c}
E_{n} \\
D_{n} X_{n}+F_{n}
\end{array}\right)=\left(\begin{array}{c}
P_{n} \\
Q_{n}
\end{array}\right) R_{n} .
$$


QR with Exponential Windowing

Proof. The proof is by induction. The theorem is clearly true for $n=0$ (take $P_{0}=I$ and $R_{k}=0$ ).

Now suppose that the theorem is true for some $n \geq 0$, and suppose that $R_{n}$ has been updated so that (2.2) holds. Then from (2.1) and (2.2) we have that

$$
\left\|\left(\begin{array}{c}
\beta R_{n} \\
x_{n}^{\mathrm{H}}
\end{array}\right)\right\| \leq\left\|R_{n+1}\right\|+\left\|\left(\begin{array}{c}
G_{n} \\
h_{n}^{\mathrm{H}}
\end{array}\right)\right\| \leq \rho+K \epsilon_{\mathrm{M}}\left\|\left(\begin{array}{c}
\beta R_{n} \\
x_{n}^{\mathrm{H}}
\end{array}\right)\right\| .
$$

Hence

$$
\left\|\left(\begin{array}{c}
\beta R_{n} \\
x_{n}^{\mathrm{H}}
\end{array}\right)\right\| \leq \frac{\rho}{1-K \epsilon_{\mathrm{M}}} .
$$

Now consider the equations

$$
\begin{aligned}
&\left(\begin{array}{c}
\beta E_{n}+P_{n} G_{n} \\
\beta\left(D_{n} X_{n}+F_{n}\right)+Q_{n} G_{n} \\
x_{n+1}+h_{n}^{\mathrm{H}}
\end{array}\right)=\left(\begin{array}{cc}
P_{n} & 0 \\
Q_{n} & 0 \\
0 & 1
\end{array}\right)\left(\begin{array}{c}
\beta R_{n}+G_{n} \\
x_{n+1}+h_{n}
\end{array}\right) \\
&=\left(\begin{array}{cc}
P_{n} & 0 \\
Q_{n} & 0 \\
0 & 1
\end{array}\right)\left(\begin{array}{cc}
U_{n}^{\mathrm{H}} & w_{n} \\
v_{n}^{\mathrm{H}} & \bar{\xi}_{n}
\end{array}\right)\left(\begin{array}{cc}
U_{n} & v_{n} \\
w_{n}^{\mathrm{H}} & \xi_{n}
\end{array}\right)\left(\begin{array}{c}
\beta R_{n}+G_{n} \\
x_{n+1}+h_{n}
\end{array}\right) \\
&=\left(\begin{array}{cc}
P_{n} U_{n}^{\mathrm{H}} & P_{n} w_{n} \\
Q_{n} U_{n}^{\mathrm{H}} & Q_{n} w_{n} \\
v_{n}^{\mathrm{H}} & \bar{\xi}_{n}
\end{array}\right)\left(\begin{array}{c}
R_{n+1} \\
0
\end{array}\right)
\end{aligned}
$$

They suggest that we should take

$$
\begin{aligned}
P_{n+1} & =P_{n} U_{n}^{\mathrm{H}}, \\
Q_{n+1} & =\left(\begin{array}{c}
Q_{n} U_{n}^{\mathrm{H}} \\
v_{n}^{\mathrm{H}}
\end{array}\right), \\
E_{n+1} & =\beta E_{n}+P_{n} G_{n}, \\
F_{n+1} & =\left(\begin{array}{c}
\beta F_{n}+Q_{n} G_{n} \\
h_{n}^{\mathrm{H}}
\end{array}\right) .
\end{aligned}
$$

In fact all we need do is verify that $E_{n+1}$ and $F_{n+1}$ so defined satisfy the bound (2.4). From (2.6) and the fact that (2.3) has orthonormal columns, we have

$$
\left\|\left(\begin{array}{c}
E_{n+1} \\
F_{n+1}
\end{array}\right)\right\| \leq \beta\left\|\left(\begin{array}{c}
E_{n} \\
F_{n}
\end{array}\right)\right\|+\left\|\left(\begin{array}{c}
G_{n} \\
h_{n}^{\mathrm{H}}
\end{array}\right)\right\| .
$$


Hence from the induction hypothesis (2.4) and from (2.1) and (2.5)

$$
\left\|\left(\begin{array}{c}
E_{n+1} \\
F_{n+1}
\end{array}\right)\right\| \leq \frac{\beta K \epsilon_{\mathrm{M}} \rho}{(1-\beta)\left(1-K \epsilon_{\mathrm{M}}\right)}+\frac{K \epsilon_{\mathrm{M}} \rho}{1-K \epsilon} \leq \frac{K \epsilon_{\mathrm{M}} \rho}{(1-\beta)\left(1-K \epsilon_{\mathrm{M}}\right)},
$$

which establishes the theorem.

One unsatisfactory aspect of this theorem is that it is phrased in terms of an upper-bound on the computed R-factor. This leaves open the possibility that $R_{n}$ could grow unboundedly, even though the true factors remain bounded. The following corollary shows that this cannot happen.

Corollary 2.3. Let

$$
\eta=\frac{K \epsilon_{\mathrm{M}}}{(1-\beta)\left(1-K \epsilon_{\mathrm{M}}\right)}
$$

and let $\hat{\rho}$ be an upper bound on the norms of the true $R$-factors. Then

$$
\rho \leq \frac{\hat{\rho}}{1-\eta}
$$

Proof. Since $R_{n}$ is obtained from $D_{n} X_{n}$ by a unitary transformation, $\left\|D_{n} X_{n}\right\| \leq$ $\hat{\rho}$. From Theorem 2.2, it follows that

$$
\rho \leq \hat{\rho}+\eta \rho,
$$

from which the corollary follows immediately.

There are three comments to make about this theorem. First, the bound says that the combined effect of all the operations is the same as if we had introduced a relative perturbation in $D X_{n}$ of approximately norm $\eta \cong-K \epsilon_{\mathrm{M}} /(1-\beta)$. For example, if $\beta=0.5$, the effect of all the updates is only twice the effect of a single update whatever the value of $n$. Thus, there is never a need to restart the computation to get rid of accumulated rounding errors.

Second, we have focused on the QR factorization for the sake of simplicity. However, the analysis applies mutatis mutandis to more complicated decomposition such as the URV and ULV decomposition [2,3], in which unitary transformations are applied to both sides of $D_{n} X_{n}$. The key is to observe that the updating algorithms have backward error analyses in the spirit of Theorem 2.1.

Finally, as we mentioned in the introduction, the bounds are likely to overestimate the error in the long run. The errors do not spread evenly over $D_{n} X_{n}$, as the bound seems to imply, but tend to decrease exponentially along with the rows of $D_{n} X_{n}$. We will now proceed to analyze this phenomenon. 
QR with Exponential Windowing

\section{Exponential Decay of the Error}

The reason for the weakness of the bounds derived in the last section is that we have ignored the structure of $Q_{n}$ in passing from the recurrence

$$
F_{n+1}=\left(\begin{array}{c}
\beta F_{n}+Q_{n} G_{n} \\
h_{n}^{\mathrm{H}}
\end{array}\right)
$$

to a bound on the backward error. It turns out that the rows of $Q_{n}$ can decrease exponentially at approximately the same rate as the rows of $D_{n} X_{n}$. When this fact is taken into account, we obtain a more realistic bound for the old data.

Theorem 3.1. Let

$$
\xi \geq\left\|x_{i}^{\mathrm{H}}\right\|
$$

be an upper bound on the norms of the $x_{i}^{\mathrm{H}}$ and let

$$
\rho^{(-1)} \geq\left\|R_{i}^{-1}\right\|, \quad i=p, p+1, \ldots,
$$

be an upper bound on the norms of the inverses of the computed $R_{i}$. Let

$$
\begin{aligned}
& \kappa=\rho \rho^{(-1)}, \\
& \tau=\frac{K \epsilon_{\mathrm{M}}}{1-K \epsilon_{\mathrm{M}}}, \\
& \tilde{\beta}=\beta+\kappa \tau .
\end{aligned}
$$

Then if $f_{i n}^{\mathrm{H}}$ denotes the backward error in the ith row of $D X_{n}$,

$$
\left\|f_{i n}^{\mathrm{H}}\right\| \leq \tilde{\beta}^{n-i} \tau(\rho+(n-i) \kappa \xi), \quad i=p, p+1 \ldots ; n=i, i+1, \ldots .
$$

Proof. Let $q_{i n}^{\mathrm{H}}$ denote the $i$ th row of $Q_{n}$. Then

$$
\beta^{n-i} x_{i}^{\mathrm{H}}+f_{i n}^{\mathrm{H}}=q_{i n}^{\mathrm{H}} R_{n} .
$$

Hence

$$
\left\|q_{i n}^{\mathrm{H}}\right\| \leq \rho^{(-1)}\left(\beta^{n-i} \xi+\left\|f_{i n}^{\mathrm{H}}\right\|\right) .
$$

Now from (2.6),

$$
\begin{aligned}
& f_{i i}=h_{i}^{\mathrm{H}}, \\
& f_{i, n+1}^{\mathrm{H}}=\beta f_{i n}^{\mathrm{H}}+q_{i n}^{\mathrm{H}} G_{n}, \quad n=i, i+1, \ldots .
\end{aligned}
$$


Hence from the bound on $\left\|G_{n}\right\|$ developed in the proof of Theorem 2.2 and (3.1) we have

$$
\begin{aligned}
\left\|f_{i i}^{\mathrm{H}}\right\| & \leq \tau \rho, \\
\left\|f_{i, n+1}^{\mathrm{H}}\right\| & \leq \tilde{\beta}\left\|f_{i n}^{\mathrm{H}}\right\|+\tilde{\beta}^{n-i} \kappa \tau \xi, \quad n=i, i+1, \ldots
\end{aligned}
$$

Hence if we set

$$
\begin{aligned}
\varphi_{i i} & =\tau \rho, \\
\varphi_{i, n+1} & =\tilde{\beta} \varphi_{i n}+\tilde{\beta}^{n-i} \kappa \tau \xi, \quad n=i, i+1, \ldots,
\end{aligned}
$$

then $\left\|f_{i n}^{\mathrm{H}}\right\| \leq \varphi_{\text {in }}$. But it is easily verified that

$$
\varphi_{i n}=\tilde{\beta}^{n-i} \tau(\rho+(n-i) \kappa \xi) .
$$

The proof of the theorem must be modified for the case $i<n$, since in this case $R_{i}$ is singular. The key is to use the bound from Theorem 2.2 as an initial condition for the recursion (3.3). The resulting bound exhibits the same exponential decay.

The number $\kappa$ is an upper bound on the condition of the $R_{n}$, and if some of the $R_{n}$ are very ill-conditioned, the bounds will be large. However, note that even in applications in which rank-degenerate $R_{n}$ are expected (e.g., direction of arrival estimation), the presence of noise in the data is likely to make the ill-conditioning very mild compared with the rounding unit.

Finally, note that because of the presence of the term $(n-1) \kappa \xi$, the bounds of this section are initially weaker that the bounds of the preceding section. However, as $n$ increases these bounds ultimately become sharper, since they track the decreasing error while the bounds of the preceding section remain constant.

\section{References}

[1] G. H. Golub and C. F. Van Loan (1989). Matrix Computations. Johns Hopkins University Press, Baltimore, Maryland, 2nd edition.

[2] G. W. Stewart (1990). "An Updating Algorithm for Subspace Tracking." Technical Report CS-TR 2494, Department of Computer Science, University of Maryland. To appear in IEEE Transactions on Signal Processing.

[3] G. W. Stewart (1991). "Updating a Rank-Revealing ULV Decomposition." Technical Report CS-TR 2627, Department of Computer Science, University of Maryland. 
[4] J. H. Wilkinson (1965). The Algebraic Eigenvalue Problem. Clarendon Press, Oxford, England. 